\title{
LA FLORICULTURA VALENCIANA ANTE EL RETO EUROPEO
}

\author{
José Daniel Gómez López
}

\section{RESUMEN}

El presente articulo analiza el desarrollo del cultivo de flores y planta ornamental en la Comunidad Valenciana y particularmente en la provincia de Alicante.

El incremento gradual de la producción de flores se debe en gran medida a las especiales condiciones climáticas existentes en la región, lo que permite cualitativamente y cuantitativamente competir con otros países productores en la Comunidad Económica Europea.

No obstante, la problemática existente en torno a la sobreexplotación del agua, los escasos recursos financieros destinados al desarrollo de la investigación agrobiológica y la excesiva parcelación de las propiedades con la consecuente atomización de la oferta, son algunos de los problemas a los que se enfrenta la floricultura valenciana ante el ingreso en la Comunidad Económica Europea.

\begin{abstract}
The present article analyze development of the flowers and ornamental plants cultivation in the Valenciana Conmunity, and Alicante province particulary.

The gradual increment of the flowers production is consequence of special climatology in the region, that permit contend quantitative and qualitatively with others producers countries of the European Economic Conmunity.

Although, the problem existent about the great consuption of water, scarces financials recourses for develop the agrobiology investigation and the excessive parcellong of properties, with the increment of offer, these are some problemes that have the valenciana floriculture front the ingress into European Economic Conmunity.
\end{abstract}

\section{RESUMO}

O presente artigo analiza o desenvolvimento do cultivo de flores e plantas ornamentais da Comunidade Valenciana e particularmente da provincia de Alicante.

$\mathrm{O}$ incremento gradual da produçao de flores é devida em grande medida ás especiais condiçoes climatológicas existentes na regiao o que permite competir qualitativamente e quantitativamente com outros países da Comunidade Económica Europeia. 
Nao obstante, a problemática existente em torno á sobreexploraçao da agua, os escasos recursos financeiros destinados ao desenvolvimento da pesquisa agrobiológica e a excesiva parcelaçao da propriedade com a consequente diversificaçao da oferta, sao alguns dos problemas a que enfrenta-se a floricultura valenciana no seu ingresso na Comunidade Económica Europeia.

\section{Introducción}

A fines del siglo XIX y comienzos del XX, entre la alta sociedad se incrementa el consumo de productos derivados de la jardinería. Numerosos tratados y libros son escritos por aficionados y expertos cultivadores de flores, para incrementar y promocionar el cultivo más científicamente, a partir de la observación de experiencias extranjeras. Cabe destacar el Tratado completo del cultivo de huerta, que introduce en su estudio "el cultivo forzado o de lujo, esto es, los invernaderos especialmente utilizados en jardinería" 1 .

La evolución del cultivo de flores en la Comunidad Valenciana se ve interrumpido por la Guerra Civil, que tuvo consecuencias negativas en la evolución de la floricultura pues ya existía una pequeña infraestructura en el desarrollo de nuevas variedades y en el uso de nuevas técnicas de cultivo. Una vez finalizada la contienda, la recuperación productiva es paulatina y se efectúa en áreas muy específicas, especialmente en los alrededores de grandes núcleos urbanos, donde la existencia de una demanda muy puntual y en fechas muy señaladas, permite la supervivencia de pequeños agricultores dedicados a este cultivo.

Posteriormente, debido a los efectos de la Segunda Guerra Mundial, la producción y el consumo de flores sufre una recesión generalizada a nivel mundial y, especialmente europeo. El cultivo de flores y plantas ornamentales está íntimamente asociado al ascenso de los niveles de vida. En el período de postguerra, aquél se verá afectado no solamente en Europa, sino también en España, donde además de un incipiente consumo comenzaba a estructurarse una prometedora corriente exportadora de flores.

Con la recuperación económica de Europa, existe una verdadera eclosión de cultivos de flores y plantas ornamentales debido en cierta medida a un deseo manifiesto de olvidar la guerra que alcanza a España a partir de los años sesenta. Este despertar en España del cultivo y producción de flores para su comercialización, traerá como consecuencia una excesiva atomización de las explotaciones agrícolas lo que, en muchos casos, servirá para obtener una baja rentabilidad.

Además de problemas estructurales, el incremento de la producción de flores ocasionará un descuido en la elección de nuevas variedades, permitiendo la proliferación y transmisión de virosis entre los cultivos, ocasionando un bajo rendimiento cualitativo del producto. En cuanto a la comercialización, el exceso de oferta y una estabilización de la demanda, contribuyeron a un descenso generalizado del precio del producto, incidiendo directamente en las explotaciones con el consecuente abandono de éstas o su transformación varietal.

A partir de mediados de los años setenta y especialmente a principios de los años ochenta, el sector comienza a recuperarse bajo un nuevo planteamiento. La introducción y mejora varietal traerá como consecuencia la adaptación biológica de flores al clima mediterráneo, consiguiendo variedades más resistentes a la virosis, lo que incidirá directamente en un 
incremento substancial de la producción y de la calidad. Asimismo, la introducción y uso de nuevas tecnologías ha permitido avanzar en el uso de nuevos materiales de riego adaptados a las necesidades de la región, lo que permite un ahorro en agua sin limitar la producción. Fruto de esa labor de investigación, lo constituye el cultivo hidropónico actualmente aplicado a la floricultura que consigue resultados espectaculares.

En la actualidad, el sector de flores para corte y plantas ornamentales en España y, especialmente en la Comunidad Valenciana, se prepara para ese gran reto que constituye el entrar en el mercado europeo compitiendo directamente con las empresas gigantes en el sector como son las holandesas y francesas especialmente.

\section{La luminosidad, base de la competitividad en la Comunidad Valenciana}

Uno de los factores que más beneficia el cultivo y producción de flores y plantas ornamentales en la Comunidad Valenciana, es su clima y, dentro del clima, la luminosidad o número de horas de sol.

Las condiciones climáticas de la región valenciana son realmente excepcionales en relación a muchas Comunidades del territorio nacional y, particularmente, a los países comunitarios europeos. El número de horas de sol de la Comunidad Valenciana en verano es uno de los más elevados que se observan en Europa, y en los meses de octubre a marzo, la insolación también contempla índices superiores a la Europa comunitaria.

La Comunidad Valenciana se encuentra atravesada por las isohelias de 1.107 horas en Alicante; 1.062 en Castellón y 991 horas en Valencia. Es decir, que estas condiciones permiten al agricultor valenciano en principio, una economía de inputs energéticos de la que carece el agricultor europeo, el cual se ve obligado a transferir al valor final del producto el sobregasto ocasionado por la utilización de energía lo cual incrementa notablemente el producto haciendolo poco competitivo.

Los agricultores dedicados al cultivo de flores en invernadero, saben que entre los factores de producción, la luz o luminosidad es uno de los más difíciles de proporcionar. Se consigue equilibrar rentablemente los fertilizantes, agua, etc., y, gracias al material de recubrimiento del invernadero, se consigue asimismo una mejoría en el balance térmico natural. No obstante, en el cultivo de ciertas variedades de flores para corte, se requieren altos índices de luminosidad para la obtención de un excelente producto y, hasta ahora no ha sido posible incrementar la luminosidad artificialmente sin incidir en el coste final del producto. La insolación y consecuentemente la luminosidad existente en el litoral mediterráneo español, ha permitido que la producción de flores irrumpa con éxito en el mercado europeo.

Estos datos adquieren mayor relevancia puesto que pertenecen a meses en que la insolación es muy limitada y, cuando la luz solar es imprescindible para los cultivos fuera de temporada. En el próximo cuadro se puede observar la insolación existente en algunas ciudades europeas de diferentes latitudes, pero que tienen en común que en los países donde se encuentran tienen un importante sector de floricultura. Asimismo hay que señalar la importancia de los meses comprendidos entre el otoño y el invierno para el comercio de flores, ya que, la falta de oferta y un incremento en la demanda permiten obtener unos precios sensiblemente elevados.

Haciendo abstracción de los meses comprendidos entre la primavera y el verano, agrupando los comprendidos entre octubre y marzo y comparándolos con las horas de insolación durante esos meses en Alicante, obtenemos: 
Cuadro I

PROMEDIO DE HORAS DE SOL Y ENERGIA TÉRMICA DE INCIDENCIA FN KCALIMETRO CUADRADOIHORA EN LOS MESES DE OCTUBRE A MARZO EN LA COMUNIDAD VALENCIANA

\begin{tabular}{lccccccccccccc}
\hline \multicolumn{1}{c}{ PROVINCIA } & \multicolumn{1}{c}{ OCTUBRE } & \multicolumn{2}{c}{ NOVIEMBRE } & \multicolumn{2}{c}{ DICIEMBRE } & \multicolumn{2}{c}{ ENERO } & \multicolumn{2}{c}{ FEBRERO } & \multicolumn{2}{c}{ MARZO } & TOTAL \\
\hline & Horas sol & Kcal. & Horas sol & Kcal. & Horas sol & Kcal. & Horas sol & Kcal, & Horas sol & Kcal. & Horas sol & Kcal. & Horas sol \\
\hline Alicante & 216 & 185.760 & 186 & 159.960 & 171 & 147.060 & 182 & 156.520 & 190 & 163.400 & 213 & 183.180 & 1.158 \\
Castellón & 198 & 170.280 & 173 & 148.780 & 158 & 135.880 & 171 & 147.080 & 170 & 146.200 & 204 & 175.440 & 1.074 \\
Valencia & 197 & 169.420 & 151 & 129.860 & 142 & 122.120 & 157 & 135.020 & 168 & 144.480 & 190 & 163.400 & 1.005 \\
\hline
\end{tabular}

Fuente: García-Badell, J.: La energía, el hombre y la agricultura 1979, pp. 45.

Cuadro II

INSOLACIÓN MENSUAL EN DIFERENTES CIUDADES DE LA EUROPA COMUNITARIA (media 1956-70) en horas

\begin{tabular}{lccccc}
\hline MESES & $\begin{array}{c}\text { VLISSINGEN } \\
\text { (Holanda) }\end{array}$ & $\begin{array}{c}\text { PERPIGNAN } \\
\text { (Francia) }\end{array}$ & $\begin{array}{c}\text { MARSELLA } \\
\text { (Francia) }\end{array}$ & $\begin{array}{c}\text { BRUSELAS } \\
\text { (Belgica) }\end{array}$ & $\begin{array}{c}\text { ALMERí } \\
\text { (España) }\end{array}$ \\
\hline Enero & 54 & 161 & 134 & 54 & 189 \\
Febrero & 70 & 172 & 157 & 77 & 190 \\
Marzo & 134 & 209 & 208 & 119 & 227 \\
Abril & 169 & 245 & 251 & 158 & 309 \\
Mayo & 216 & 255 & 281 & 129 & 331 \\
Junio & 232 & 279 & 323 & 196 & 362 \\
Julio & 210 & 322 & 368 & 198 & 264 \\
Agosto & 200 & 282 & 324 & 198 & 227 \\
Septiembre & 152 & 236 & 253 & 160 & 185 \\
Octubre & 104 & 189 & 191 & 110 & 173 \\
Noviembre & 51 & 158 & 151 & 36 & 3.053 \\
Diciembre & 39 & 136 & 123 & 1.585 & \\
\hline Total & 1.629 & 2.644 & 2.764 & & \\
\hline
\end{tabular}

Fuente: World Survey of climatology. 1970. Elaboración propia. 
Cuadro III

INSOLACIÓN EXISTENTE ENTRE OCTUBRE-MARZO EN DIRERENTES CIUDADES EUROPEAS (en horas)

\begin{tabular}{lrrr}
\hline CIUDAD & PAÍS & PERÍODO & $\begin{array}{c}\text { HORAS } \\
\text { INSOLACIÓN }\end{array}$ \\
\hline Vlissingen & Holanda & octubre-marzo & 452 \\
Perpignam & Francia & octubre-marzo & 1.025 \\
Marsella & Francia & octubre-marzo & 964 \\
Bruselas & Bélgica & octubre-marzo & 456 \\
Almería & España & octubre-marzo & 1.191 \\
Alicante & España & octubre-marzo & 1.158 \\
\hline
\end{tabular}

Fuente: World Survey of Climatology, 1970. Anuario de Estadística Agraria M.A.P.A. Varios años. Elaboración Propia.

\section{El agua, factor de altas inversiones tecnológicas}

El agua es sin duda uno de los factores limitativos de la expansión del cultivo de invernadero en general y de la floricultura en particular, especialmente en provincias como Alicante, donde existe un gran déficit de la misma. El agua además de beneficiar el cultivo, es un importante regulador de las temperaturas de las plantas, manteniéndolas en equilibrio constante e impidiendo las bruscas variaciones térmicas que se producen en el vegetal, especialmente tratándose de un cultivo tan sensiblemente como son las flores.

Mediante el riego por goteo se aporta agua y nutrientes al suelo, poniéndolos a disposición de la zona radicular de la planta, en la misma proporción que la pierde por evapotranspiración ${ }^{2}$. El riego por goteo o fertirriego es quizá la técnica más empleada en los cultivos de flor cortada y planta ornamental -sin excluir otros como el riego por aspersión, etc.No obstante, el alto costo de instalación y mantenimiento hace que su utilización en explotaciones de tipo familiar esté muy limitada, permitiendo a las empresas tecnológicamente más equipadas incrementar notablemente la disparidad productiva y cualitativa ya existente.

El clavel, cultivo muy extendido en la Comunidad Valenciana y especialmente en la provincia de Alicante, requiere riegos abundantes durante su plantación, y posteriormente durante el enraizamiento de los esquejes. Una disminución en el suministro de agua motivaría irremediablemente un decrécimo de su rendimiento productivo, repercutiendo directamente en la calidad y duración media del producto, una vez comercializado.

La utilización de agua con un alto índice de salinidad repercutiría negativamente en la calidad de la flor y en su rendimiento productivo, disminuyendo el número de flores por unidad de superficie. Los estudios técnicos actualmente intentan actuar cada vez más sobre los sistemas de riego, y utilización de aguas, permitiendo que éstos, además de atender a una necesidad imperiosa de economizar, sirvan para utilizar aguas con un alto contenido de sales, sin alterar el equilibrio biológico del vegetal.

2 RINCÓN SÁNCHEZ, L., Riegos localizados en cultivos horticolas. Murcia, Dpto. Hortofruticultura. INIACRIDA. 
Cuadro IV

CONSUMO MENSUAL DE AGUA DEL CLAVEL AMERICANO EN INVERNADERO

\begin{tabular}{lcrr}
\hline MES & $\begin{array}{c}\text { NECESIDAD DE AGUA } \\
\text { (L-METRO CUADRADO) }\end{array}$ & MES & $\begin{array}{r}\text { NECESIDAD DE AGUA } \\
\text { (L-METRO CUADRADO) }\end{array}$ \\
\hline Enero & 30 & Julio & 200 \\
Febrero & 45 & Agosto & 150 \\
Marzo & 90 & Septiembre & 100 \\
Abril & 135 & Octubre & 60 \\
Mayo & 180 & Noviembre & 45 \\
Junio & 225 & Diciembre & 30 \\
\hline & & Total & 1.290 \\
\hline
\end{tabular}

Fuente: GUERRERO, I.: El cultivo rentable de las flores. 1987 pp. 40.

\section{Localización geográfica de los cultivos florales}

Debido a ser un cultivo destinado preferentemente a una demanda de los grandes núcleos urbanos o a su exportación extraprovincial, las explotaciones de flor cortada y planta ornamental se localizan frecuentemente en las proximidades de las ciudades, o donde existan vías de comunicación que permitan un acceso rápido al mercado. No obstante, la localización también está sujeta o dependerá en gran medida del factor climático, instalándose donde exista una máxima insolación invernal, factor fundamental en este tipo de cultivo.

La provincia de Valencia, es la mayor productora de flor cortada y planta ornamental $-45,4$ y 67,8 por ciento respectivamente- de la Comunidad Valenciana (figs. 1 y 2). Los viveros se localizan esencialmente en los alrededores de la capital. Las comarcas de la Horta Sud, Horta Nord, La Ribera y La Safor, ocupan un lugar destacado en cuanto a producción de planta ornamental y flor cortada al aire libre.

Castellón es la provincia menos desarrollada en este tipo de cultivo - 0,6 y 9,2 por ciento en flor cortada y planta ornamental respectivamente-. Los viveros también se localizan en las proximidades de la capital y en algunos núcleos del litoral como Benicarló. Alicante ocupa un segundo puesto en volumen productivo en flor cortada y planta ornamental -54 y 23 por ciento respectivamente-. No obstante, ocupa un primer lugar en cuanto a producción de variedades de flor cortada más comercializada en el exterior del país. Destaca el cultivo de clavel y rosa, dos variedades muy apreciadas en los mercados europeos y en la que la provincia de Alicante ocupa el primer puesto en producción dentro de la Comunidad Valenciana. Asimismo, es la primera provincia productora de orquídeas y otras variedades de flor cortada menos comercializadas. La distribución de las empresas productoras y viveristas en la provincia de Alicante está muy localizada. Existen tres grandes zonas productoras: el Pilar de la Horadada, Campo de Elche y el Medio Vinalopó. Por especies, cabe destacar en planta ornamental el Campo de Elche, Campo de Alicante, Marina Alta y Medio Vinalopó. En cuanto a flor cortada, su localización se concentra en el Pilar de la Horadada, Campo de Elche, Campo de Alicante y Medio Vinalopó.

\section{La estructura productiva principal handicap al desarrollo de la floricultura valenciana}

Las fuentes utilizadas en este estudio son el Censo Agrario de 1982, elaborado por el 


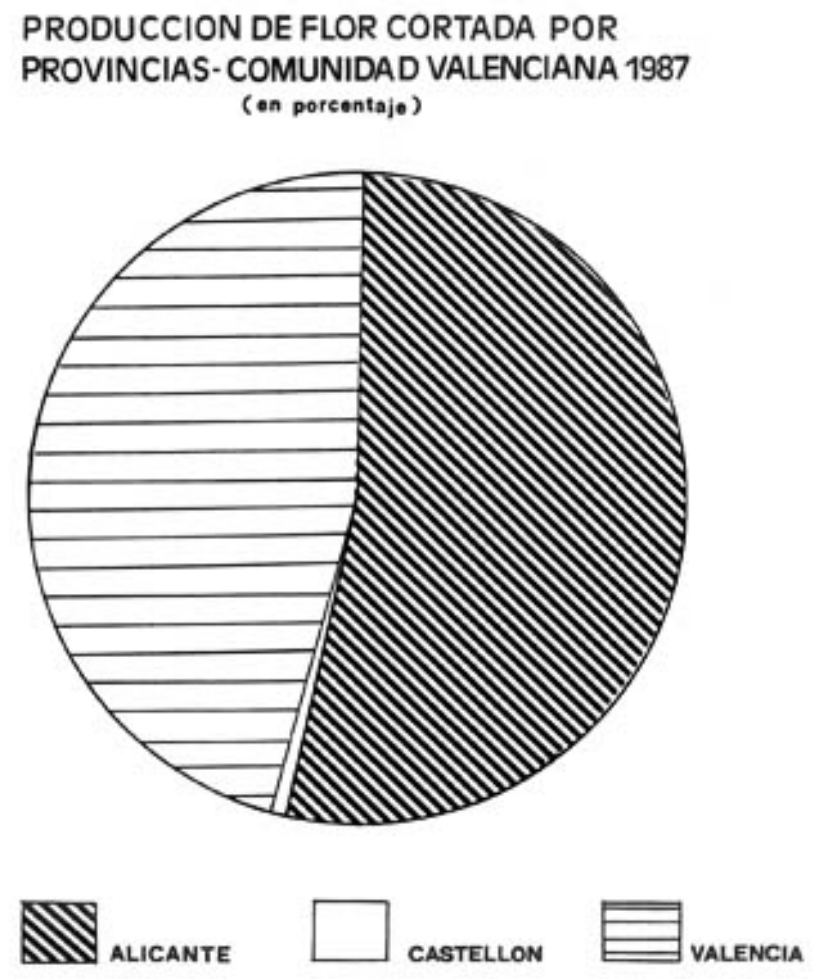

FIGURA 1

Instituto Nacional de Estadística, y el Catálogo de Flores y Plantas Ornamentales, editado en 1987 por la Consellería de Agricultura y Pesca de la Generalitat Valenciana. De acuerdo con esta última publicación, la superficie total ocupada por el cultivo de flor cortada y planta ornamental en la Comunidad Valenciana ascendía en 1987 a 730 hectáreas. De éstas, 550 hectáreas eran de cultivos al aire libre y 180 hectáreas de cultivo protegido; las últimas se distribuyen en 100 hectáreas de flor cortada y 80 hectáreas de planta ornamental.

Del total porcentual de la superficie ocupada por el cultivo de flor cortada bajo abrigo en la Comunidad Valenciana, la provincia de Valencia ocupa un 53,8 por ciento, seguida de Alicante con un 44,2 por ciento y por último Castellón con un 2 por ciento. Cabe destacar dentro de la superficie protegida, que el apartado referente a los invernaderos sin calefacción, donde la provincia de Alicante ocupa una superficie expresada del 53,2 por ciento en flor cortada, seguida de Valencia y Castellón con un 44,5 y 2,3 por ciento respectivamente. Este hecho adquiere mayor relevancia al comparar los costes internos de producción entre las distintas provincias, que favorece a la provincia de Alicante en la relación precio-coste, lo que permite una mayor intensificación productiva al existir un ahorro en inputs.

Por otro lado, una economía en la utilización de los inputs energéticos, permite una mayor movilidad y reempleo del capital ya que, no se invierten fuertes sumas en factores fijos de capital productivo.

En cuanto a la superficie ocupada por las explotaciones destinadas al cultivo de flor cortada y planta ornamental en la Comunidad Valenciana, hay que remitirse al Censo Agrario de 1982. La superficie media de una explotación de flor cortada en el conjunto de la 


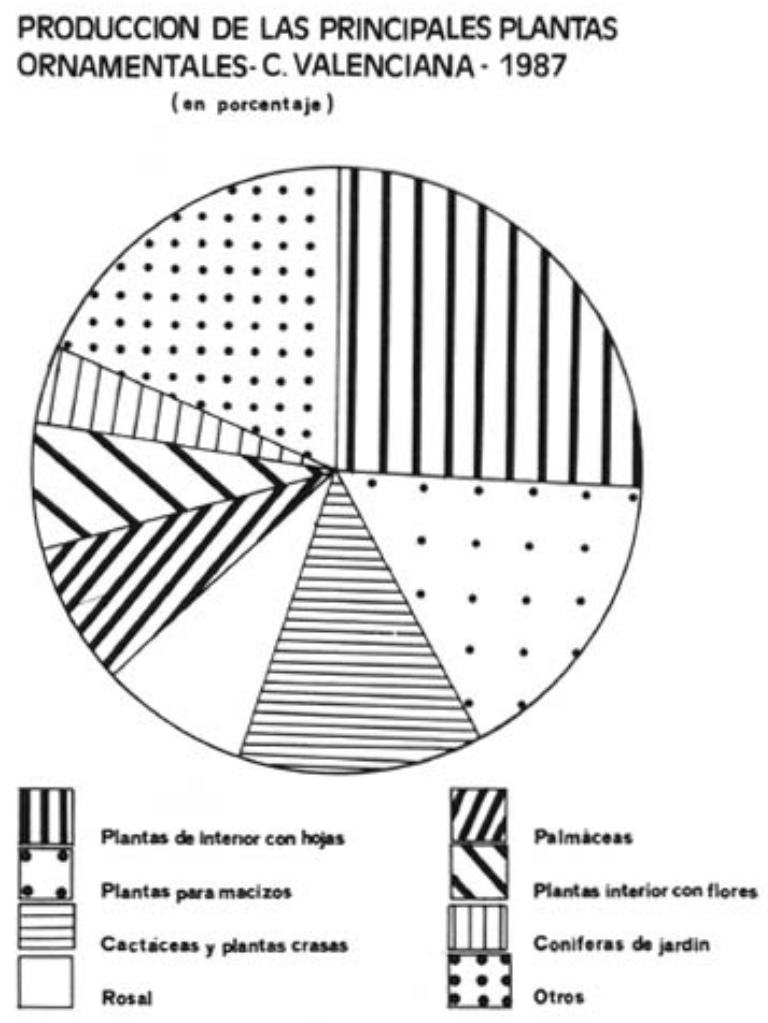

FIGURA 2

Comunidad Valenciana considerada de carácter familiar, se sitúa entre los 3.000 y 5.000 metros cuadrados aproximadamente. Atendiendo a esta especificidad, la Comunidad Valenciana mantiene globalmente una estructura atomizada. De un total de 733 explotaciones en regadío censadas en 1982 en la Comunidad Valenciana, 476 son menores de 3 hectáreas, ocupando una superficie de 171 hectáreas de un total absoluto de 483 hectáreas. Existen también 240 explotaciones que ocupan una superficie de 202 hectáreas, con una dimensión comprendida entre las 3 y 30 hectáreas. Finalmente, las 17 restantes explotaciones ocupan 112 hectáreas, con una extensión comprendida entre las 300 y 1.000 hectáreas.

Por otro lado, en el Censo Agrario de 1982, por primera vez se contemplaron además de las explotaciones en regadío, los invernaderos. Los datos referentes a estas últimas explotaciones bajo abrigo, constituye a nuestro entender, el elemento más preciso en la caracterización del cultivo de flores, ya que, en el marco de una estructura productiva de carácter comercial, el cultivo bajo abrigo permite mantener una producción escalonada para el abastecimiento de un mercado muy puntual y localizado. De este modo, el cultivo bajo abrigo es la expresión física de una evolución cualitativa y selectiva de la producción de flores para corte.

En el Censo Agrario de 1982 se contabilizaron un total de 99 invernaderos o explotaciones bajo abrigo en la Comunidad Valenciana, distribuidos por provincias de la siguiente forma: Castellón, 13 explotaciones bajo abrigo; Valencia, 49 y Alicante 37 invernaderos. La 
Cuadro V

SUPERFICIE DE VIVEROS Y FLOR CORTADA

COMUNIDAD VALENCIANA-1987 (en metros cuadrados)

\begin{tabular}{|c|c|c|c|}
\hline & ALICANTE & CASTELLÓN & VALENCIA \\
\hline \multicolumn{4}{|l|}{ AIRE LIBRE } \\
\hline -Ornamental & 1.350 .000 & 300.000 & 3.500 .000 \\
\hline -Flor Cortada & 140.000 & 60.500 & 306.500 \\
\hline - Total & 1.490 .500 & 360.500 & 3.806 .500 \\
\hline \multicolumn{4}{|c|}{ SUPERFICIE PROTEGIDA } \\
\hline -Ornamental & 58.000 & 5.000 & 128.000 \\
\hline —Flor Cortada & 8.500 & 1.000 & 38.500 \\
\hline \multicolumn{4}{|c|}{ INVERNADERO SIN CALEFACCIÓN } \\
\hline -Ornamental & 75.000 & 700 & 174.000 \\
\hline —Flor Cortada & 348.500 & 15.000 & 292.000 \\
\hline \multicolumn{4}{|c|}{ INVERNADERO CON CALEFACCIÓN } \\
\hline -Ornamental & 28.000 & 22.300 & 175.000 \\
\hline -Flor Cortada & 62.500 & 4.000 & 180.500 \\
\hline -Total & 580.500 & 48.000 & 988.000 \\
\hline TOTAL GENERAL & 2.071 .000 & 408.500 & 4.794 .500 \\
\hline
\end{tabular}

Fuente: Consellería d'Agricultura i Pesca, 1987. Elaboración propia.

superficie total ocupada por los invernaderos en las tres provincias, era de 988.822 metros cuadrados o casi 99 hectáreas que, desglosado por provincias, arroja esta superficie: Castellón 39.056 metros cuadrados; Valencia 674.607 metros cuadrados y Alicante 275.159 metros cuadrados. La relación invernaderos/superficie, daba una media para toda la Comunidad Valenciana de 9.988 metros cuadrados por explotación. No obstante, las cifras correspondientes a cada provincia señalan que las explotaciones bajo abrigo en Castellón tienen una dimensión media de 3.000 metros cuadrados, lo que la sitúa en el límite de lo que se ha considerado como una explotación de carácter familiar, que está comprendida globalmente entre los 3.000 y 5.000 metros cuadrados. En la provincia de Valencia, la superficie media es de casi 14.000 metros cuadrados y en Alicante de 7.500 metros cuadrados.

Tomando como referencia la explotación familiar -en la que el componente laboral lo constituye preferentemente la mano de obra familiar, pudiendo ocasionalmente ésta ser incrementada por trabajo temporal-, con la superficie delimitada entre 0,3 y 0,5 hectáreas, de las 13 explotaciones o invernaderos existentes en Castellón en 1982, el 84,6 por ciento son de tipo familiar y a veces tienen una superficie que se caracteriza por ser un microminifundio. El 15,4 por ciento restantes pueden ser consideradas como explotaciones medias por su superficie.

La situación en la provincia de Valencia ofrece otra variable estructural, especialmente en cuanto a una clara diferenciación en las inversiones de trabajo y capital. La mitad de las 49 explotaciones bajo abrigo tensadas, eran de carácter familiar, mientras que el 47 por ciento podrían considerarse como explotaciones medias y, el 4 por ciento restante como grandes explotaciones, donde existen recursos en tierra y trabajo suficientes para incrementar el rendimiento físico por unidad de superficie. 
En la provincia de Alicante, de un total de 37 invernaderos censados en 1982, el 70 por ciento eran de tipo familiar. El 24 por ciento de tipo medio y el 6 por ciento por grandes empresas agrícolas -la cifra más alta de la Comunidad-.

La tendencia verificada en el Censo Agrario de 1982, en cuanto a la distribución de la superficie ocupada por las explotaciones bajo abrigo en las tres provincias de la Comunidad Valenciana, se mantiene en lo esencial en 1987. A través del Catálogo de Flores y Plantas Ornamentales y, atendiendo a la producción de flor cortada, se ha elaborado el próximo cuadro, pero antes haremos una aclaración. En el Catálogo de flores para corte la relación producción/superficie viene expresada en metros cuadrados/unidades. En la elaboración de este cuadro se ha transformado la variable unidades por una unidad más utilizada en los censos de producción referentes a producción de flores: docenas.

\begin{tabular}{|c|c|c|c|c|}
\hline \multicolumn{5}{|c|}{$\begin{array}{c}\text { Cuadro VI } \\
\text { PRODUCCIÓN DE FLOR CORTADA DE ACUERDO A LA SUPERFICIE DE } \\
\text { EXPLOTACIÓN(docenas-metros cuadrados) }\end{array}$} \\
\hline PRODUCCIÓN & SUPERFICIE & ALICANTE & CASTELLÓN & VALENCIA \\
\hline más de 834 & $0-42$ & 5 & 2 & 8 \\
\hline $835-4.167$ & $43-208$ & - & 1 & 13 \\
\hline $4.168-16.667$ & $209-833$ & 8 & 2 & 7 \\
\hline $16.668-41.667$ & $834-2.083$ & 12 & - & 8 \\
\hline $41.668-83.334$ & $2.084-4.167$ & 8 & - & 5 \\
\hline $83.335-166.667$ & $4.168-8.333$ & 7 & - & 2 \\
\hline más de 166.668 & más de 8.334 & 3 & - & 3 \\
\hline
\end{tabular}

Fuente: Consellería d'Agricultura. Elaboración propia.

Para la elaboración del cuadro anteriormente expuesto, se ha tomado como referencia el cultivo del clavel fue ocupa una superficie del 71 por ciento en 1987 en la Comunidad Valenciana- (figs. 3 y 4). Haciendo abstracción de las posibles disparidades existentes en cuanto a productividad por explotación y variedad por provincias y, admitiendo una productividad media de 20 docenas por metro cuadrado -la productividad generalmente oscila entre las $13 \mathrm{y}$ 25 docenas $^{3}$ y las 18 a 20 docenas $^{4}$ por metro cuadrado- se obtendría teóricamente las cifras representadas en el cuadro.

En la provincia de Alicante, de los 43 floricultores censados en 1987, el 56 por ciento se pueden caracterizar por su nivel productivo y la superficie ocupada, como explotación de tipo familiar. El 35 por ciento como explotación media y, el 9 por ciento restante como empresa altamente capitalizada, donde el empleo de altos porcentajes de inputs productivos permiten mantener un ciclo productivo de tipo intensivo.

Castellón de un total de 5 floricultores censados en 1987, el 100 por ciento son considerados como explotación de carácter familiar. Por último, en la provincia de Valencia de un total censado en 1987 de 46 floricultores, el 78 por ciento son por el volumen cosechado, explotaciones familiares, el 16 por ciento explotaciones medias y un 6 por ciento grandes empresas.

3 ZAPATA NICOLAS, M.; MARTÍNEZ ROMERO. G., Los sistemas de producción en invernadero en la región del Segura. Murcia, ed. Regional, 1981, 315 pp.

4 PALOMAR OVIEDO, F., Nuevas Técnicas en horticultura. Almería. Ed. Cajalmería, 1988, 347 pp. 


\section{PRODUCCION DE LAS PRINCIPALES FLORES \\ C. VALENCIANA-1987 (en porcentaje)}

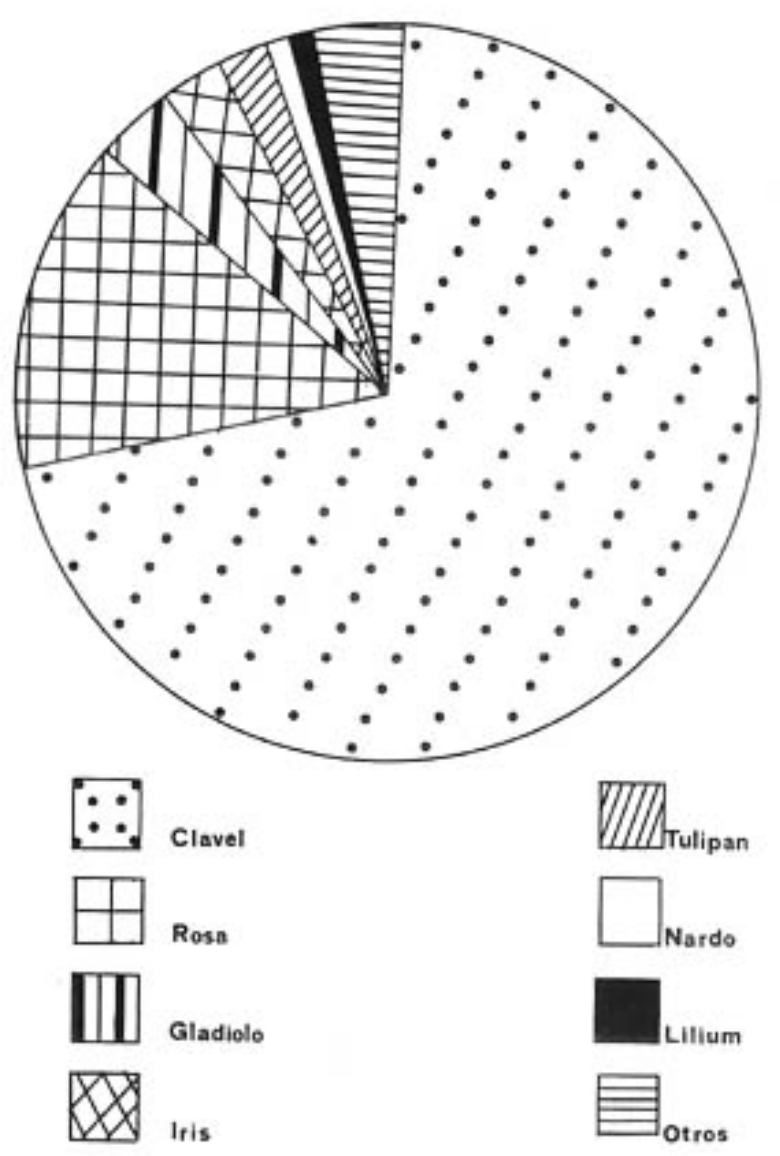

FIGURA 3

La tendencia verificada en cuanto a la producción de las explotaciones, viene íntimamente asociada al destino final de la misma (figura 1). Generalmente, la producción de las explotaciones de tipo familiar se comercializa en un radio próximo a donde está situado físicamente la misma. Cuando se encuentra próxima a los centros urbanos, el propio agricultor es el que comercializa su producto en los Mercados Centrales. Si la explotación está situada, a distancia de un núcleo urbano, la producción es vendida a un intermediario.

Las explotaciones con un volumen productivo medio cuentan con más canales de comercialización, al obtener un producto cualitativamente más favorable a la demanda. Muchos de estos floricultores, están integrados en una red distributiva extraprovincial, lo que favorece sensiblemente la relación precio-coste. Por último, las grandes empresas además de comercializar su producto en el mercado interno nacional, una parte muy importante del volumen productivo se exporta a los mercados generalmente europeos. Son empresas in- 


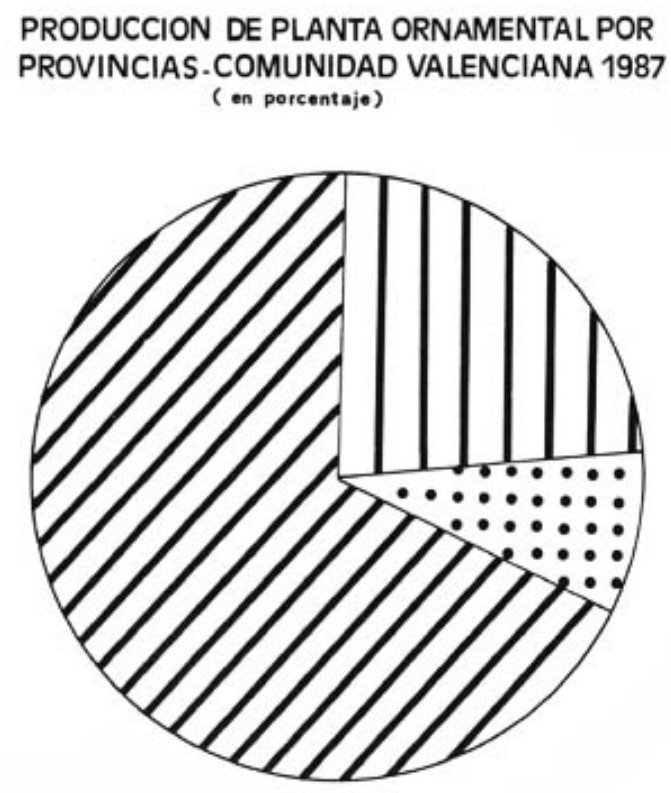

IIIII ALCANTE

$\because::$

$\mathbb{Z I I}_{\text {vatencen }}$

FIGURA 4

tegradas verticalmente y casi siempre forman parte de una red comercializadora extendida por todo el mundo.

El destino final de la producción valenciana de flor para corte se distribuye así: (figura 5) la provincia de Alicante cuenta porcentualmente con el índice más elevado de empresas exportadoras hacia Europa; la producción de la provincia de Valencia se destina, en primer lugar, a su comercialización interprovincial y después al mercado extraprovincial; Castellón prácticamente comercializa su producción dentro de la propia Comunidad.

En relación a la planta ornamental, el destino final de su producción analizado por provincias, ofrece un cuadro bastante diferenciado al de la flor cortada. En principio, la provincia de Valencia ocupa un primer puesto en cuanto a producción y comercialización, así como al número de empresas existentes en este sector productivo. De un total de 163 viveristas censados en la Comunidad Valenciana en 1987, el 70 por ciento estaban establecidos en la provincia de Valencia; el 21 por ciento en la de Alicante y un 9 por ciento en Castellón.

La exportación y comercialización interna se reparte como indica el cuadro 8.

\section{EI reto europeo}

En el tratado de Adhesión de España a la Comunidad Económica Europea, no se contempla a los productos derivados de la floricultura como sensibles, es decir, no están sujetos a barreras arancelarias ni a restricciones cuantitativas, lo cual en principio permite una expansión del sector que únicamente estará limitada por el propio sistema de regularización 

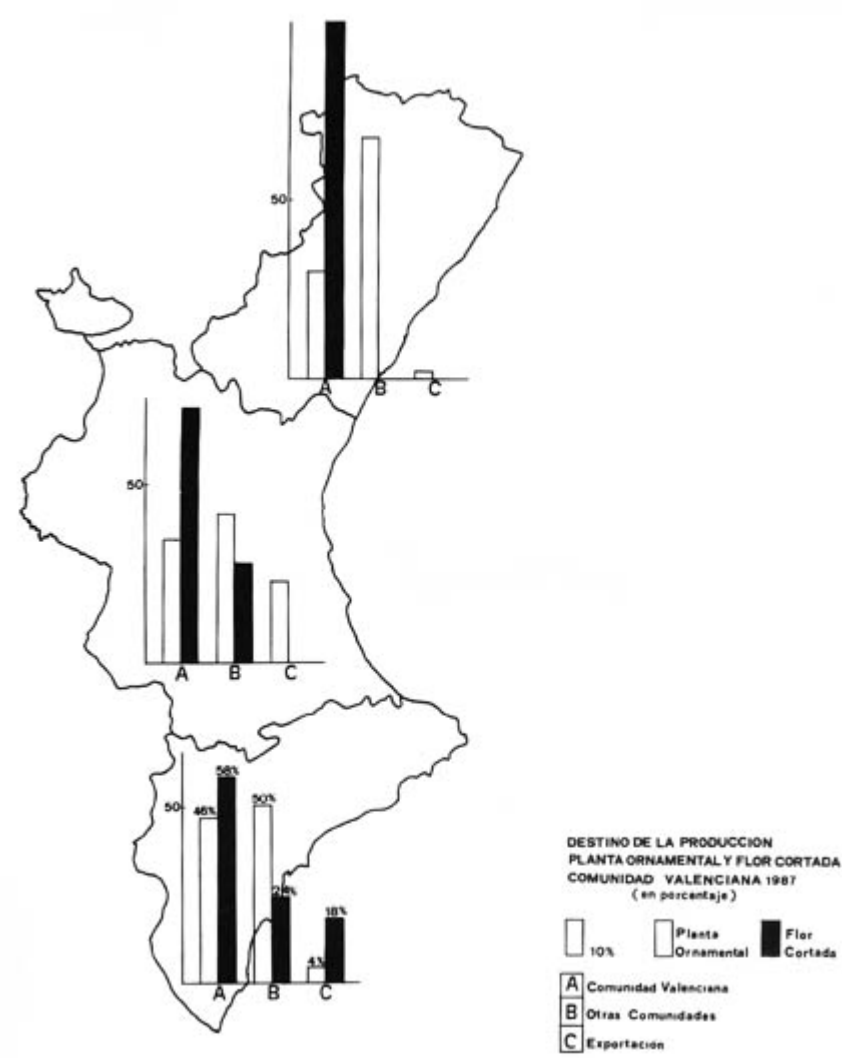

FIGURA 5

de la competencia en los mercados comunitarios. Atendiendo a un posible incremento del volumen exportado hacia estos mercados de la Europa Comunitaria, las grandes empresas productoras españolas y valencianas en particular, están llevando a cabo una labor intensa que permita concentrarla oferta y controlar todo el ciclo productivo de la flor cortada y planta ornamental. Esta labor se realiza tomando como perspectiva la integración plena en un mercado pleno, lo que supondrá hacer frente a la dura competencia de empresas europeas sobre todo holandesas y francesas.

En la Comunidad Valenciana, la concentración productiva se manifiesta más palpablemente en el subsector flor cortada que en el de planta ornamental, quizás debido a que el uso de nuevas tecnologías -factor básico en el desarrollo de una gran empresa- es más accesible a la gran explotación que a la explotación de tipo familiar. En planta ornamental los mayores viveristas de la Comunidad Valenciana obtienen el 30 por ciento de la pro- 
Cuadro VII

DESTINO DE LA PRODUCCIÓN DE FLOR CORTADA

COMUNIDAD VALENCIANA-1987 (en porcentaje)

\begin{tabular}{lcccc}
\hline DESTINo & ALICANTE & CASTELLÓN & VALENCIA & TOTAL \\
Comunidad Valenciana & 58 & 100 & 72 & 64 \\
Otras Comunidades & 24 & - & 28 & 26 \\
Exportación & 18 & - & - & 18 \\
\hline
\end{tabular}

Fuente: Consellería d'Agricultura.

Cuadro VIII

DESTINO DE LA PRODUCCIÓN DE PLANTA ORNAMENTAL COMUNIDAD VALENCIANA-1987 (en porcentaje)

\begin{tabular}{lcccc}
\hline DESTINo & ALICANTE & CASTELLÓN & VALENCIA & TOTAL \\
\hline Comunidad Valenciana & 46 & 30 & 35 & 35 \\
Otras Comunidades & 50 & 68 & 42 & 46 \\
Exportación & 4 & 2 & 23 & 19 \\
\hline
\end{tabular}

Fuente: Consellería d'Agricultura.

ducción, mientras que en flor cortada los mayores floricultores obtienen el 50 por ciento de la producción y ocupan el 42 por ciento de la superficie destinada al cultivo ${ }^{5}$.

$\mathrm{La}$ inexistencia de rígidas barreras aduaneras que regule el mercado de flores en la Europa comunitaria, no impide que ésta utilice una serie de instrumentos que dan cuerpo al Reglamento 234/68 que establece un sistema basado en un conjunto de normas calidad. Estas normas son en realidad parámetros por los cuales se establece un precio base para las distintas variedades de flores y dentro de éstas las diferentes categorías. Las normas de calidad hacen referencia a la variedad y, especialmente a la composición física del producto -longitud de tallo, grado de curvatura del mismo, resistencia, modificación del tallo, etc.- y la coloración de la flor. En muchos mercados es práctica habitual que las grandes empresas a través de esos agentes para evitar la competencia exterior exigen unas normas de calidad muy rígidas.

En la etapa actual, el sector va a estar sometido a una transición de siete años lo mismo que la mayoría de los productos agrícolas españoles. Además de los posibles beneficios fiscales que se deriven de la plena integración en la Comunidad Económica Europea existe para las empresas productoras valencianas, la posibilidad de participar del mercado potencialmente más importante del mundo, el Mercado Europeo.

El alto nivel de renta y el hábito adquirido por sus poblaciones de consumir flores, permiten obtener unas cifras de consumo per cápita en 1985 de 9.000 ptas./año de flores en Holanda -la más alta del mundo- seguida de Alemania Federal, Bélgica, Suecia y Noruega. Como se puede apreciar, de los cinco países mayores consumidores de flores, en su totalidad son europeos y de éstos, tres pertenecen a la Europa Comunitaria.

El reto del sector de la floricultura y planta ornamental valenciana, lo constituye la

5 CONSELLERÍA DE AGRICULTURA. Catálogo de flores y plantas ornamentales. Generalitat Valenciana, 1987. 62 pp. 
adaptabilidad de las empresas grandes y familiares a la innovación y utilización tecnológica. Holanda y Francia son países donde el sector productivo y comercial se encuentra muy desarrollado gracias a las inversiones realizadas en la investigación biológica vegetal para la obtención de nuevas variedades así como a una amplia labor de marketing. Las empresas de estos países ya se están preparando para instalarse en España, principalmente en provincias donde exista una tradición floral -entre ellas la Comunidad Valenciana-, beneficiándose de la integración y aprovechando la disponibilidad de inputs energéticos a bajo costo.

La necesidad de aplicar fuertes cantidades de capital es básico para la supervivencia y expansión del sector, debido a que el empleo de nuevas tecnologías implica el uso de nuevas maquinarias, así como de material de propagación y vegetativo, vital para un desarrollo eficaz de la competitividad comercial. La potenciación de la investigación debe partir conjuntamente de empresarios y organismos oficiales, lo que permitirá obtener las propias variedades cultivadas en la Comunidad Valenciana sin tener que depender del suministro exterior.

\section{Bibliografía}

La información obtenida se debe en gran parte a la labor de campo efectuada en zonas productoras de flores, especialmente en empresas exportadoras y en otras donde su comercialización se procesa en la propia Comunidad Valenciana. También se ha utilizado como base de apoyo a la elaboración del presente artículo los siguientes estudios:

GARCÍA BADELL, J., La energía solar, el hombre y la agricultura, Madrid. Ministerio de Agricultura, 1982. WAA., World Surrey of Climatology. London, New York, Paris, 1970.

GUERRERO, Isabel, El cultivo rentable de las flores, Barcelona. Ed. del Vecchi. 1987, 158 pp. 